\title{
Demand response in adjustment markets for electricity*
}

\author{
Claude Crampes ${ }^{\dagger}$ and Thomas-Olivier Léautier ${ }^{\ddagger}$
}

January 18, 2015

\begin{abstract}
This article examines the participation of consumers in adjustment markets for electricity power. These markets allow market participants to respond to random supply shocks occurring after quantities have been contracted. Under perfect competition, opening the adjustment market to consumers always increase ex post efficiency, hence welfare, as expected. However, this result is not robust to strategic behavior by consumers who hold private information on their value for electricity power. We prove that under such information asymmetry, allowing consumers to enter the adjustment market may reduce welfare. This arises because suppliers limit the information rents they must abandon by proposing inefficient $e x$ ante retail contracts. If the value of $e x$ post efficiency gains due to consumers' participation is low, whereas the information distortion is high, the overall net effect is a welfare decrease.
\end{abstract}

Keywords: electricity consumption, adjustment market, demand response, information asymmetry

JEL codes: D11, D21, Q41

\footnotetext{
${ }^{*}$ The authors thank two anonymous referees of the journal who have suggested important changes in the paper presentation.

${ }^{\dagger}$ Toulouse School of Economics (GREMAQ and IDEI), claude.crampes@tse-fr.eu

${ }^{\ddagger}$ Toulouse School of Economics (IAE, IDEI, CRM), thomas.leautier@tse-fr.eu
} 


\section{Introduction}

Historically, electricity demand was not responsive to variations in wholesale prices. As liquid wholesale markets for electric power did not exist in most countries until the early $1990 s$, there was no price signal for users to respond to. Furthermore, users were accustomed to consume power to respond to their needs, not to the opportunity cost of power at any given time. Over the last 20 years, demand response has become a technical reality. Liquid wholesale markets now exist in most countries, providing the value of power at different times and locations. Most large industrial and commercial users have meters that enable them and their supplier to track their real time consumption. Similar "smart"meters are being deployed for residential and professional users in most jurisdictions.

Demand response has the potential to transform how power systems are designed and operated. For example, if enough users reduce their demand as price increases, there may no longer be a need to implement involuntary curtailment, hence the notion of capacity adequacy, which currently stands at the core of most electric power markets' design, may no longer be relevant. For this reason, demand response has received significant attention from academics and policy makers in Europe and North America ${ }^{1}$.

In most industries, consumers respond to demand by purchasing on the spot market and adjusting their consumption to the spot price. The electricity industry is different, since most consumers have fixed-price contracts with their supplier, which specify a price independent of the spot price. Rather than reacting to real time price, demand response is thus customers reselling into an adjustment market the Megawatt-hours they are entitled to withdraw ${ }^{2}$.

The first issue that has attracted attention of academics and policy makers is the design of adjustment markets, in particular the price customers must pay to have access to the market and the price they receive when re-

\footnotetext{
${ }^{1}$ See in particular Borenstein et al. (2002) and Hogan (2009).

${ }^{2}$ Demand response is not demand management. In the latter, consumers buy electricity power at low price because they accept the risk to be disconnected by their provider. Ex post, they are not the decision maker. Oren (2013) presents a rich historical perspective of this type of arrangement. Interruptible contracts can be promoted by means of option mechanisms, as shown in Kamat \& Oren (2002). Demand response is "A reduction in the consumption of electric energy by retail customers from their expected consumption in response to an increase in the price of electric energy or to incentive payments designed to induce lower consumption of electric energy." (USCA, 2012b).
} 
selling power. As discussed in Section 5, it has led to contentious debates in many jurisdictions ${ }^{3}$.

Even if the adjustment market is well-designed, a second issue of interest is consumers' potential strategic behavior. Customers are paid for the difference between the consumption-that-would-have-happened, called their baseline, and their actual consumption. They thus have incentives to inflate that baseline. In a particularly illuminating example, in June 2013, the Federal Energy Regulatory Commission fined a demand response operator for wrongly claiming, on behalf of its client the Maryland Stadium Authority, that it reduced its electricity usage from the baseline in 2009 and 2010 at the main Baltimore baseball stadium. Specifically, the enquiry established that the operator's engineers turned on the lights at the baseball stadium to create false demand that it was then paid to reduce ${ }^{4}$.

To address this issue, a first possibility is to develop algorithms that provide a robust estimation of the baseline. While this approach may work for some customers, it may not apply to all. Another approach is to recognize that some consumers will always have better information than retailers on their baseline, hence to design contracts that explicitly acknowledge this private information. This article is the first to do so, i.e. to explicitly model consumers' strategic behavior using contract theory ${ }^{5}$. As will be discussed below, the analysis yields new and unexpected results.

The situation we examine is as follows. Ex ante, suppliers and consumers agree on bilateral supply contracts. Then, a random shock occurs. Demand and supply must then be rebalanced ex post, i.e., after the shock is realized. We examine the impact of introducing an adjustment market on both ex post and ex ante efficiency, i.e., we compare outcomes when compensation for the shock is provided solely by increased production, and when it is provided by an adjustment market in which producers and customers participate.

The analysis incorporates two realistic features of the electric power industry. First, customers have private information on their value for electricity, which gives them the opportunity to behave strategically. Second, contrary to most papers dealing with demand curtailment, the contract price is not given by a condition of free entry with a uniform price inefficiently

\footnotetext{
${ }^{3}$ See in particular Ruff (2002) and USCA (2012a, 2012b, 2014).

${ }^{4}$ http://www.theenergyfix.com/2013/07/25/two-ferc-settlements-illustrate-attemptsto-game-demand-response-programs/\#sthash.AdXi8tK9.dpbs

${ }^{5}$ See Laffont and Martimort (2002).
} 
fixed at the average cost of electricity. Instead, it arises endogenously form the bargaining between retailers and consumers.

Standard economic analysis suggests that, if the adjustment market is well-designed and frictionless, resale is ex post efficient: consumers resell power precisely up to the point where their marginal value for power equals the price in the adjustment market ${ }^{6}$. Furthermore, if there are no frictions in the ex ante retail market, ex post demand response does not distort the ex ante contract either, which is then ex ante efficient. In other words, neither customers nor suppliers have an incentive to distort the ex ante contract from the efficient one when consumers are allowed to intervene in the adjustment process. Opening the adjustment market to customers thus increases welfare. This intuition is confirmed by the analysis, and summarized in Lemma 1.

However, the result does not necessarily hold under imperfect information. Suppose there are two types of customers. Contract theory suggests suppliers must leave an information rent to customers with the higher willingness-to-pay (high type), and reduce consumption of the low type customers. We prove that, while the ex ante contract remains efficient for high type customers, it is no longer efficient for low type. Thus, the possibility of customers' strategic behavior, which gives rise to the information rent, defeats ex ante efficiency of adjustment markets (Proposition 1).

Finally, we examine the net welfare impact of customers participation in the adjustment market. We prove that, in some instances, the information distortion for low type customers is so large that opening the adjustment market to customers reduces welfare (Proposition 2).

The policy implication of our work is not that policy makers should abandon adjustment markets for fear of inefficiency. Rather policy makers and regulators ought to be aware that consumers will exert strategic behavior when possible, and should therefore design markets that accommodate this strategic behavior.

The paper is organized as follows. Section 2 introduces the notations and presents the perfect information benchmark. Section 3 discusses the case of asymmetric information. Section 4 analyses the welfare impact of opening the adjustment market to customers on welfare. In Section 5 we

\footnotetext{
${ }^{6}$ See for example Chao (2010) and Crampes and Léautier (2012).
} 
derive some lessons for regulators. Section 6 concludes.

\section{The benchmark model}

We consider a two-stage game. In the first stage, each customer (she) faces a supplier (he) who offers her a contract, i.e., a pair (quantity of $k W h$, payment). ${ }^{7}$ The customer accepts or rejects the offer, depending on her characteristics and outside options.

In the second stage, there are two possible states of nature: either $(i)$ all generation units are operational so that the contracts signed during stage 1 are implemented, or $(i i)$ some units are out of order. In this case, an adjustment market is opened where producers with generation reserves and customers holding withdrawal rights compete. Adjusted contracts are then implemented.

We first specify the model and then determine the retail and adjustment equilibria under symmetric information.

\subsection{Setup and notation}

Suppliers This article assumes that electricity suppliers are in exclusive bilateral negotiations with their customers in the contract market, while the adjustment market (described later) is perfectly competitive. This is the case if incumbent retailers have de facto monopoly in their (former) service territory, while the adjustment market covers multiple service territories, which seems to be a reasonable approximation in many North American and European markets. First, empirical evidence suggests that electricity customers are reluctant to switch suppliers. ${ }^{8}$ Assuming that incumbent retailers engage in exclusive bilateral negotiations with their customers in the contract market is a reasonable approximation of the contracting stage. Second, in most US markets, the geographical footprint of the Independent

\footnotetext{
${ }^{7}$ In most countries we know, consumers pay a flat unit price per $\mathrm{kWh}$, and they are limited in their instantaneous demand by the capacity of their meters. Along the day, the week, the month, consumption varies so that the bill varies. In the paper, to keep things simple, we consider a one period model, or equivalently, a multiperiod model with stationary needs. Then, consumers are able to decide on the total quantity they want to consume and the total expenditure they are ready to pay. In a slightly more complex model, the value attribute of the contract could be the unit price instead of the total payment.

${ }^{8}$ See Deller et al. 2014, and Wilson and Waddams (2012).
} 
System Operator, which administers the adjustment market, encompasses the service territories of a dozen or more utilities. Similarly in Great Britain, the dispatch is national, while at the "big six" retailers are descendants of the twelve Regional Electricity Company.

Each supplier offers a representative consumer a contract $(q, t)$, where $q$ is the quantity and $t$ the total payment. If the consumer rejects the offer, she receives net utility $U$, and the supplier receives no profit. The contract is further specified below.

Producers The suppliers are vertically integrated into production. This is a reasonable assumption, as many suppliers produce most of the energy they sell. The marginal cost of producing output $x$ is denoted $c(x)$, and is assumed to be non decreasing, $c^{\prime}(x) \geq 0$.

Consumers Consumers' surplus is $S(q, \theta)$, where $q$ is the quantity consumed and $\theta$ the consumer's type. The function $S(q, \theta)$ is increasing and concave in $q$, increasing in $\theta$ and satisfies the Spence-Mirrlees condition (Marginal Rate of Substitution increasing in type):

$$
S_{q \theta}(q, \theta) \equiv \frac{\partial^{2} S(q, \theta)}{\partial q \partial \theta}>0
$$

Consumers know better than suppliers their value for power, i.e., $\theta$ is consumer's private information.

Production outage and adjustment After contracting has taken place between customers and suppliers and before delivery, a random shock occurs, that may reduce production ${ }^{9}$. The shock is $\mu \geq 0$, distributed according to the density function $f($.$) on [0, K]$, where $K$ is exogenous. The probability that actual production is equal to contracted production is $\operatorname{Pr}(\mu=0)=$ $(1-\beta)>0$.

We assume the shock $\mu>0$ can always be compensated, i.e., there is enough production reserve and demand response to balance supply and demand in the adjustment market. Without loss of generality, we suppose

\footnotetext{
${ }^{9}$ Note that the need for rebalancing could come from a unexpected increase in consumption. Consumers with low utility could also participate efficiently in the adjustment process.
} 
that the outage affects the least expensive production units. ${ }^{10}$ Then, ex post the total cost to produce quantity $q$ when outage is $\mu$ is

$$
C(q, \mu)=\int_{\mu}^{\mu+q} c(x) d x
$$

and the marginal cost of production is

$$
\frac{\partial C(q, \mu)}{\partial q}=c(\mu+q) .
$$

Ex ante, the expected total cost of producing $q$ is

$$
\mathbb{E}_{\mu}[C(q, \mu)]=(1-\beta) C(q, 0)+\beta \int_{0}^{K} C(q, \mu) f(\mu) d \mu,
$$

and the expected marginal cost is

$$
\mathbb{E}_{\mu}[c(q+\mu)]=(1-\beta) c(q)+\beta \int_{0}^{K} c(q+\mu) f(\mu) d \mu .
$$

We assume the adjustment market is perfectly competitive: a large number of producers/suppliers and customers (when allowed) are price takers when bidding produced or curtailed volumes.

\subsection{Perfect information benchmark}

In this section, we suppose that each supplier knows perfectly the characteristic of each of his customers, $\theta$.

\subsubsection{No demand participation in adjustment}

Consider first the situation where suppliers/producers must balance the electric system without the customers' help. When failure occurs they have no choice but to turn on more expensive production units to meet their commitments. If supplier $i$ suffers shortage $\mu_{i}$ after contracting on $\left(t_{i}, q_{i}\right)$, he produces the missing quantity by using his own reserve or buys from the adjustment market at price $p_{a}$, where other producers will sell additional production.

\footnotetext{
${ }^{10}$ For a given size of the shortage $\mu$, the ranking of the missing plants in the merit order is indifferent. The marginal cost is just "shifted to the left" by $\mu$.
} 
Since the adjustment market is assumed to be perfectly competitive, $p_{a}$ is equal to marginal cost $c(q+\mu)$. We assume that all producers are hit by an identical shock. Thus, when customers are not allowed to bid, the adjustment is neutral in terms of income for producers, since there is no trade to compensate $\mu$ since each is producing with his own reserves. Consequently, the ex ante expected profit of any supplier is simply $\left(t-\mathbb{E}_{\mu}[C(q, \mu)]\right)$.

At the contracting stage, the supplier maximizes his profit, under the constraint that the customer's net utility exceeds her reservation utility $U$. This leads the supplier to maximize the net surplus from the transaction, i.e., to set $t=S(q(\theta), \theta)+U$ and then to choose $q$ that solves

$$
\max _{q} S(q, \theta)-\mathbb{E}_{\mu}[C(q, \mu)]
$$

If suppliers had less market power, they would would leave a positive rent to customers, determined in equilibrium (see Stole, 2007). This would lead to much more complex modelling, but would not modify much the main insights of this article. We thus make the simplifying assumption that the rent left to consumers is constant. Without loss of generality, we normalize it to zero.

The quantity produced and sold is $q(\theta)$ determined by the first-order condition

$$
P(q(\theta), \theta)=\mathbb{E}_{\mu}[c(q(\theta)+\mu)],
$$

where $P \stackrel{\text { def }}{=} S_{q}$ is the inverse demand function.

Since the ex ante contract is designed taking into account the expected shock, it is almost never ex post efficient. More precisely, denoting $q^{*}(\theta, \mu)$ the ex post efficient production and $\hat{\mu}$ the production shock such that $c\left(q^{*}(\theta, \hat{\mu})+\hat{\mu}\right)=$ $\mathbb{E}_{\mu}[c(q(\theta)+\mu)]$, we show in Appendix A that contracted production is lower than ex post optimal production for a small production shock $(\mu<\hat{\mu})$. Conversely, contracted production is too high for a large production shock $(\mu>\hat{\mu})$.

\subsubsection{Demand participation in adjustment}

Suppose now customers can reduce their demand to help balancing the production shortage $\mu$. Specifically, after she has paid $t$ to buy $q$, customer $\theta$ can resell a share $q_{a}^{c}$ on the adjustment market at unit price $p_{a}$. Produc- 
ers also participate in the adjustment market by selling $q_{a}^{s}$ at unit price $p_{a}$. Neither $q_{a}^{c}$ nor $p_{a}$ is under the control of suppliers. The former is chosen by consumers, the latter is determined by competition on the adjustment market.

We solve the problem backwards, examining first the ex post adjustment and second the ex ante retail contract.

Equilibrium in the adjustment market If there is an energy shortage $\mu>0$, given the contracted quantity $q$ and the price $p_{a}$ on the adjustment market, consumer $\theta$ solves

$$
\begin{gathered}
\max _{q_{a}^{c}} S\left(q-q_{a}^{c}, \theta\right)+p_{a} q_{a}^{c} \\
\text { s.t. } q_{a}^{c} \leq q
\end{gathered}
$$

Assuming an interior solution, the first-order condition is

$$
p_{a}-P\left(q-q_{a}^{c}, \theta\right)=0 \Leftrightarrow \widetilde{q}_{a}^{c}\left(p_{a}, q, \theta\right)=q-D\left(p_{a}, \theta\right),
$$

where $D\left(p_{a}, \theta\right)$ is the quantity consumed by $\theta$ given the adjustment price $p_{a}$.

The adjustment market determines the net consumption $\left(q-\widetilde{q}_{a}^{c}\right)$. Quantities $q$ and $\widetilde{q}_{a}^{c}$ are complements: an increase in $q$ increases $\widetilde{q}_{a}^{c}$ one-for-one $\left(\frac{\partial \widetilde{q}_{a}^{c}}{\partial q}=1\right)$. This is a consequence of the fact that consumers pay for the entire quantity they contract ex ante $(q)$, and not only for their net ex post consumption $\left(q-\widetilde{q}_{a}^{c}\right)$.

Symmetrically, producers solve

$$
\max _{q_{a}^{s}} p_{a} q_{a}^{s}-\int_{\mu}^{q+q_{a}^{s}} c(x) d x
$$

Assuming an interior solution, the first-order condition is

$$
p_{a}-c\left(q+q_{a}^{s}\right)=0 \Leftrightarrow \widetilde{q}_{a}^{s}\left(p_{a}, q\right)=\gamma\left(p_{a}\right)-q
$$

where $\gamma()=.c^{-1}($.$) . Quantities contracted and quantities in the adjust-$ ment market are substitutes: an increase in $q$ decreases $\widetilde{q}_{a}^{s}$ one-for-one $\left(\frac{\partial \widetilde{q}_{a}^{s}}{\partial q}=-1\right)$. 
The wholesale equilibrium price is the solution to

$$
\widetilde{q}_{a}^{c}\left(p_{a}, q, \theta\right)+\widetilde{q}_{a}^{s}\left(p_{a}, q\right)=\mu \Leftrightarrow \gamma\left(p_{a}\right)-D\left(p_{a}, \theta\right)=\mu .
$$

For a given $\mu$, the equilibrium price is $p_{a}(\mu, \theta)$, increasing in both arguments ${ }^{11}$. A large $\theta$ means strong willingness to consume, thus a low willingness to sell on the wholesale market, pushing the adjustment equilibrium price upwards. Also observe that the price in the adjustment market does not depend on the contracted quantity $q$.

At equilibrium, offers are

$$
q_{a}^{c}(\mu, q, \theta) \stackrel{\text { def }}{=} \widetilde{q}_{a}^{c}\left(p_{a}(\mu, \theta), q, \theta\right)=q-D\left(p_{a}(\mu, \theta), \theta\right)
$$

by curtailed consumers, and

$$
q_{a}^{s}(\mu, q, \theta) \stackrel{\text { def }}{=} \widetilde{q}_{a}^{s}\left(p_{a}(\mu, \theta), q\right)=\gamma\left(p_{a}(\mu, \theta)\right)-q
$$

by reserve providers.

Equilibrium in the retail market The expected gross surplus of consumer type $\theta$ who contracts $q$ in the retail market, and sells $q_{a}^{c}(\mu, q, \theta)$ at price $p_{a}(\mu, \theta)$ in the adjustment market is

$$
u(q, \theta)=\mathbb{E}_{\mu}\left[S\left(q-q_{a}^{c}(\mu, q, \theta), \theta\right)+p_{a}(\mu, \theta) q_{a}^{c}(\mu, q, \theta)\right] .
$$

The consumer pays $t$ for the entire quantity $q$. This then gives her the right to sell back $q_{a}^{c}$ at price $p_{a}$.

For future reference, note that

$$
\begin{aligned}
\frac{\partial u}{\partial q} & =\mathbb{E}_{\mu}\left[P\left(q-q_{a}^{c}(\mu, q, \theta), \theta\right)\left(1-\frac{\partial q_{a}^{c}}{\partial q}\right)+p_{a}(\mu, \theta) \frac{\partial q_{a}^{c}}{\partial q}\right] \\
& =(1-\beta) P(q, \theta)+\beta \mathbb{E}_{\mu>0}\left[p_{a}(\mu, \theta)\right]
\end{aligned}
$$

since $q_{a}^{c}=0$ for $\mu=0$, which occurs with probability $(1-\beta)$, and $\frac{\partial q_{a}^{c}}{\partial q}=1$ for $\mu>0$. Increasing $q$ generates marginal gross surplus $P(q, \theta)$ if no failure occurs. It has no impact on the surplus realized in the adjustment market, since consumption $D\left(p_{a}, \theta\right)=q-\widetilde{q}_{a}^{c}\left(p_{a}, q, \theta\right)$ depends solely on the price

\footnotetext{
${ }^{11}$ See the proof in Appendix B.
} 
in the adjustment market, and not on contracted purchases $q$. Thus, if a failure occurs, the only impact of purchasing an additional unit is enabling the customer to resell it at price $p_{a}(\mu, \theta)$ in the adjustment market.

As before, the supplier chooses $t(\theta)=u(q(\theta), \theta)$, hence expected profit from type $\theta$ is

$\pi(q, \theta)=u(q, \theta)+\mathbb{E}_{\mu}\left[p_{a}(\mu, \theta) q_{a}^{s}(\mu, q, \theta)-\int_{\mu}^{q+q_{a}^{s}(\mu, q, \theta)} c(x) d x-p_{a}(\mu, \theta) \mu\right]$.

Observe that

$$
\begin{aligned}
\frac{\partial \pi}{\partial q} & =\frac{\partial u}{\partial q}+\mathbb{E}_{\mu}\left[p_{a}(\mu, \theta) \frac{\partial q_{a}^{s}}{\partial q}-c\left(q+q_{a}^{s}(\mu, q, \theta)\right)\left(1+\frac{\partial q_{a}^{s}}{\partial q}\right)\right] \\
& =(1-\beta)(P(q, \theta)-c(q)) .
\end{aligned}
$$

Total production in the adjustment market $\left(q+q_{a}^{s}\right)$ is solely determined by the adjustment market price. Contracting an additional unit only prevents the producer from reselling it at price $p_{a}(\mu, \theta)$ in the adjustment market if $\mu>0$. Since the supplier internalizes the consumer's surplus, these effects cancel out. The marginal value of contracted quantity is thus solely its value if no production failure occurs, which is simply price minus marginal cost.

This analysis yields the following:

Lemma 1 Under perfect information, opening the adjustment market to customers leads to production efficiency, whether production failure occurs or not.

Proof. Since there is no imperfection in adjustment markets, they are efficient if $\mu>0$ by the first welfare theorem. If $\mu=0$, no adjustment market takes place, and the production agreed-on during the retail contracting phase takes place. It is efficient since

$$
\frac{\partial \pi}{\partial q}=(1-\beta)(P(q, \theta)-c(q))=0 \Leftrightarrow P(q(\theta), \theta)=c(q(\theta)) .
$$

Lemma 1 strongly militates for opened adjustment markets. When demand cannot participate, suppliers anticipate the possibility of production 
failures, and incorporate it in their offers. Thus, consumption is (almost) never ex post efficient, either too high or too low.

Allowing demand to enter the adjustment market eliminates that inefficiency. If production failure occurs, the adjustment market is ex post efficient. Maybe more surprisingly, if no failure occurs, efficiency also arises: anticipating the impact of the adjustment market, suppliers make the optimal offer, which is realized if no adjustment occurs.

Thus, demand participation in adjustment market improves welfare under perfect information. As we will see below, this property is not guaranteed under asymmetric information.

\section{Contracts under asymmetric information}

We now assume that consumers' preference $\theta$ can take two values, $\bar{\theta}$ or $\underline{\theta}$, with $\bar{\theta}>\underline{\theta}$. Each consumer knows her type. Suppliers place probability $\alpha$ on the consumer type being $\bar{\theta}$.

\subsection{No demand participation in adjustment}

As in the previous section, each producer covering energy shortage with his own production plant, ex ante he chooses the pair of contracts $(t(\theta), q(\theta))_{\theta \in\{\bar{\theta}, \underline{\theta}\}}$ to maximize his expected profit:

$$
\max _{(t(\theta), q(\theta))_{\theta \in\{\bar{\theta}, \underline{\theta}\}}} \mathbb{E}_{\theta}\left[t(\theta)-\mathbb{E}_{\mu}[C(q(\theta), \mu)]\right]
$$

From the literature on incentives ${ }^{12}$, we know that the supplier must ensure each type is willing to accept the contract (Individual Rationality), and select the right contract (Incentive Compatibility). Standard analysis then yields payments

$$
t(\underline{\theta})=S(q(\underline{\theta}), \underline{\theta}) \text { and } t(\bar{\theta})=S(q(\bar{\theta}), \bar{\theta})-[S(q(\underline{\theta}), \bar{\theta})-S(q(\underline{\theta}), \underline{\theta})] .
$$

No rent is left to the low type customer $\underline{\theta}$, while the high type customer receives an information rent equal to the difference in surplus between her and the low type, at the low type consumption.

\footnotetext{
${ }^{12}$ See for example Laffont \& Martimort (2002).
} 
Replacing $t(\bar{\theta})$ and $t(\underline{\theta})$ by their above values in the supplier's expected profit and differentiating with respect to $q(\bar{\theta})$ and $q(\underline{\theta})$ yields:

$$
P(q(\bar{\theta}), \bar{\theta})=\mathbb{E}_{\mu}[c(q(\bar{\theta})+\mu)]
$$

and

$$
P(q(\underline{\theta}), \underline{\theta})=\mathbb{E}_{\mu}[c(q(\underline{\theta})+\mu)]+\frac{\alpha}{1-\alpha}(P(q(\underline{\theta}), \bar{\theta})-P(q(\underline{\theta}), \underline{\theta})) .
$$

We obtain the usual results of the literature on incentives:

1. there is no distortion at the top: $q(\bar{\theta})$ is such that the marginal surplus is equal to the expected marginal cost, and

2. the quantity offered to the low-type is reduced sufficiently to prevent the high-type from purchasing the low-type bundle.

The price charged to the low type is equal to the expected marginal cost plus the information distortion, which is positive by condition (1). Therefore, as in the symmetric information case, there is a " $\mu$-distortion"in all contracts, since in the absence of ex post demand adjustment, the contracted quantity $q$ must be produced.

\subsection{Demand participation in adjustment}

The equilibrium in the adjustment market is unchanged compared to the perfect information case.

Given the consumer's supply function on the wholesale market $q_{a}^{c}\left(\theta, q, p_{a}\right)$, if $q_{a}^{c}$ is observable, the producer who fixes $q$ and knows the market price $p_{a}$ can infer the value of $\theta$. However, information is revealed ex post, after the wholesale market reaches equilibrium. Ex ante, when $q$ and $t$ are fixed, the producer knows neither $q_{a}^{c}$ nor $p_{a}$. We now examine the contract market.

Surplus and profit The gross expected surplus of consumer type $\theta$ who contracts $q$ in the retail market, and will sell $q_{a}^{c}(\mu, q, \theta)$ at price $p_{a}(\mu, \theta)$ in the adjustment market is $u(q, \theta)$ defined by equation (3).

The information rent corresponding to the contracted quantity $q$ is

$$
R(q)=u(q, \bar{\theta})-u(q, \underline{\theta}) .
$$


For future reference, note that

$$
\frac{\partial R}{\partial q}=(1-\beta)(P(q, \bar{\theta})-P(q, \underline{\theta}))+\frac{\beta}{1-\beta} \mathbb{E}_{\mu>0}\left[p_{a}(\mu, \bar{\theta})-p_{a}(\mu, \underline{\theta})\right] .
$$

The marginal information rent is the sum of two terms. First, the information rent if no failure occurs $(P(q, \bar{\theta})-P(q, \underline{\theta}))$, which is the same term as without an adjustment market.

The second term is the information rent if failure occurs. This term is slightly more surprising: the previous analysis has shown that consumers behave truthfully in the adjustment market, i.e., they consume according to their type. Why should they receive a rent in the adjustment market? The answer to this apparent paradox is that the rent is received in the contract market, not in the adjustment market: anticipating they will behave truthfully in the adjustment market (hence receive no information rent), consumers require an additional rent in the contract market.

Both marginal information rents are positive. There is no a priori guarantee that the rent is higher than without an adjustment market $(P(q, \bar{\theta})-P(q, \underline{\theta}))$. However, as we will next see, the distortion is larger.

Supplier program Each producer chooses the pair of retail contracts $(t(\theta), q(\theta))_{\theta \in\{\bar{\theta}, \underline{\theta}\}}$ to maximize his expected profit:

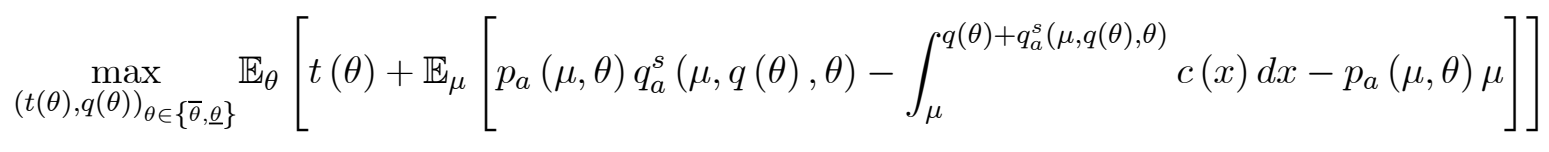

subject to individual rationality constraints

$$
u(q(\bar{\theta}), \bar{\theta})-t(\bar{\theta}) \geq 0 \text { and } u(q(\underline{\theta}), \underline{\theta})-t(\underline{\theta}) \geq 0,
$$

and incentive compatibility constraints

$u(q(\bar{\theta}), \bar{\theta})-t(\bar{\theta}) \geq u(q(\underline{\theta}), \bar{\theta})-t(\underline{\theta})$ and $u(q(\underline{\theta}), \underline{\theta})-t(\underline{\theta}) \geq u(q(\bar{\theta}), \underline{\theta})-t(\bar{\theta})$.

The Spence-Mirrlees condition holds, since

$$
\frac{\partial^{2} u}{\partial \theta \partial q}=(1-\beta) \frac{\partial P}{\partial \theta}+\beta \mathbb{E}_{\mu>0}\left[\frac{\partial p_{a}}{\partial \theta}(\mu, \theta)\right]>0
$$


by condition (1) and $\frac{\partial p_{a}}{\partial \theta}>0$.

Again, we know from the established results of incentives theory that the payment of type $\underline{\theta}$ and $\bar{\theta}$ are

$$
t(\underline{\theta})=u(q(\underline{\theta}), \underline{\theta}) \text { and } t(\bar{\theta})=u(q(\bar{\theta}), \bar{\theta})-R(q(\underline{\theta})),
$$

where $R($.$) is the information rent defined by (6).$

Producer's expected profit is

$$
\Pi=\alpha(\pi(q(\bar{\theta}), \bar{\theta})-R(q(\underline{\theta})))+(1-\alpha) \pi(q(\underline{\theta}), \underline{\theta})
$$

The first-order condition with respect to $q(\bar{\theta})$ is

$$
\frac{\partial \Pi}{\partial q(\bar{\theta})}=0 \Longrightarrow \alpha(1-\beta)(P(q(\bar{\theta}), \bar{\theta})-c(q(\bar{\theta})))=0 .
$$

Consequently the optimal quantity offered to type $\bar{\theta}$ is defined by

$$
P(q(\bar{\theta}), \bar{\theta})=c(q(\bar{\theta})) \text {. }
$$

The first order derivative with respect to $q(\underline{\theta})$ is

$$
\begin{aligned}
\frac{\partial \Pi}{\partial q(\underline{\theta})}= & (1-\alpha)(1-\beta)(P(q(\underline{\theta}), \underline{\theta})-c(q(\underline{\theta}))) \\
& -\alpha\left((1-\beta)(P(q(\underline{\theta}), \bar{\theta})-P(q(\underline{\theta}), \underline{\theta}))+\beta \mathbb{E}_{\mu>0}\left[p_{a}(\mu, \bar{\theta})-p_{a}(\mu, \underline{\theta})\right]\right) .
\end{aligned}
$$

By setting the above derivative equal to 0 , the optimal quantity offered to type $\underline{\theta}$ is implicitly defined by

$$
P(q(\underline{\theta}), \underline{\theta})=c(q(\underline{\theta}))+\frac{\alpha}{1-\alpha}\left(\begin{array}{c}
(P(q(\underline{\theta}), \bar{\theta})-P(q(\underline{\theta}), \underline{\theta})) \\
+\frac{\beta}{1-\beta} \mathbb{E}_{\mu>0}\left[p_{a}(\mu, \bar{\theta})-p_{a}(\mu, \underline{\theta})\right]
\end{array}\right)
$$

This yields the following:

Proposition 1 Suppose that customers have private information on their type. The adjustment market, if it is opened to customers, is always efficient. This efficiency translates into ex ante contract efficiency for the high-type consumer. However, the contract offered to the low type consumer is not ex ante efficient. Furthermore, the information distortion is higher than when customers do not participate in the adjustment market. 
Proof. The proposition follows immediately from the above derivations. The adjustment market when opened to customers is efficient. Equation (7) proves that the retail contract is ex ante efficient for the high type, and equation (8) proves that the retail contract is ex ante inefficient for the low type. Since $(P(q(\underline{\theta}), \bar{\theta})-P(q(\underline{\theta}), \underline{\theta}))>0$ and $\left(p_{a}(\mu, \bar{\theta})-p_{a}(\mu, \underline{\theta})\right)>0$, the total information distortion is higher than the information distortion without customers in the adjustment market, $P(q(\underline{\theta}), \bar{\theta})-P(q(\underline{\theta}), \underline{\theta})$ in equation (5).

Optimal contracting requires there is no distortion for the high type consumer. The optimal contracted quantity, presented in equation (7), is therefore ex ante efficient. If no failure occurs $(\mu=0)$, the contract is also efficient ex post.

For the low type consumer, analysis from Lemma 1 shows that increasing $q(\underline{\theta})$ has no direct impact on the supplier profits if the adjustment market is opened to customers. On the other hand, it has an impact on profits if no failure occurs (probability $(1-\beta)$ ) and on the information rent, which is proportional to $(1-\beta)$. Thus, the marginal value of $q(\underline{\theta})$ is proportional to $(1-\beta)$, and the information distortion is higher than with a limited adjustment market.

If the adjustment market accepts energy demanders, this ex ante distortion has no impact, since the market is ex post efficient. On the other hand, if no failure occurs, this inefficient retail contract is executed, which reduces welfare, as we see next.

\section{Welfare comparison}

Absent the demand side in the adjustment market, production is (almost) never optimal: too low if the production shock is small, too high otherwise. When adjustment can come from demande response, production is optimal for the high type consumer, and for the low type consumer when $\mu>0$. Only for low type consumer when $\mu=0$ is production not optimal.

One would therefore expect that opening the adjustment market increases welfare. However, this is not always the case: depending on the value of the parameters, the information distortion for low type consumers when $\mu=0$ can outweigh the gains. 
This effect is illustrated using a linear specification: marginal cost and inverse demand are linear $c(x)=c x$ with $c>0$, and $P(q, \theta)=a(\theta)-b q$, where $a(\theta)>0, b>0$, and $a^{\prime}(\theta)>0$ to respect condition (1). While a general analysis is possible, the notation would be cumbersome. The linear specification leads to simple expressions, and provides the key economic intuition.

No customer in the adjustment market Condition (4) becomes

$$
q(\bar{\theta})=\frac{a(\bar{\theta})-c \mathbb{E}[\mu]}{b+c},
$$

while condition (5) yields

$$
q(\underline{\theta})=\frac{a(\underline{\theta})-c \mathbb{E}[\mu]-\frac{\alpha}{1-\alpha}(a(\bar{\theta})-a(\underline{\theta}))}{b+c}<\frac{a(\underline{\theta})-c \mathbb{E}[\mu]}{b+c} .
$$

These expressions illustrate the two distortions discussed in this analysis. First is the production distortion: the average cost $c \mathbb{E}[\mu]$ and not the realized cost $c \mu$ is used to compute the contracted quantity. As previously mentioned, production is almost never optimal ex post. Second is the information distortion: consumption for low type consumers is further distorted by the information rent.

To guarantee that type $\underline{\theta}$ is served, we assume that

$$
a(\underline{\theta})-c \mathbb{E}[\mu] \geq \frac{\alpha}{1-\alpha}(a(\bar{\theta})-a(\underline{\theta})) .
$$

Define $y=\frac{\mathbb{E}[\mu]}{a(\underline{\theta})}$ and $x=\frac{\alpha}{1-\alpha} \frac{a(\bar{\theta})-a(\underline{\theta})}{a(\underline{\theta})}$. The variable $y$ is a measure of the production distortion, and $x$ is a measure of the information distortion. The above condition can be rewritten as

$$
a(\underline{\theta})(1-x-y) \geq 0 \Leftrightarrow(x+y) \leq 1 .
$$

Equation (9) imposes a higher bound on the sum of the two distortions.

We continue to assume that production failure when it occurs affects the least expensive units, as this leads to a simple expression of the expected net surplus: since marginal cost and inverse demand are linear, the expected 
net surplus $W^{n a}$ is a triangle. Specifically, algebraic manipulations yield

$2(b+c) W^{n a}=\mathbb{E}_{\theta}\left[(a(\theta)-c \mathbb{E}[\mu])^{2}\right]-(1-\alpha)\left((a(\underline{\theta})-c \mathbb{E}[\mu])^{2}-(a(\underline{\theta})(1-x)-c \mathbb{E}[\mu])\right)^{2}$.

Both distortions reduce welfare compared to the optimum. Standard analysis shows that the expected welfare if production was ex post optimal is $\frac{\mathbb{E}_{\theta} \mathbb{E}_{\mu}\left[(a(\theta)-c \mu)^{2}\right]}{2(b+c)}$. The production distortion reduces welfare since $(a(\theta)-c \mathbb{E}[\mu])^{2}<\mathbb{E}_{\mu}\left[(a(\theta)-c \mu)^{2}\right]$ by convexity of the function $x \mapsto x^{2}$. The information distortion reduces welfare since $(a(\underline{\theta})(1-x)-c \mathbb{E}[\mu])^{2}<$ $(a(\underline{\theta})-c \mathbb{E}[\mu])^{2}$.

If we assumed that failure could affect other units on the supply curve, equilibrium prices and quantities would not be modified, since they depend on marginal costs. On the other hand, net surplus would be higher, since more expensive units would fail. The surplus reduction from the production distorsion would be lower, which would strenghten our results, as will be shown below.

Customers in the adjustment market Price in the adjustment market $p_{a}(\mu, \theta)$ is defined by the equality of demand $\mu$ and the supply by consumers and producers

$$
p_{a}(\mu, \theta)=\frac{c}{c+b}(a(\theta)+b \mu)
$$

hence

$$
\mathbb{E}_{\mu>0}\left[p_{a}(\mu, \bar{\theta})-p_{a}(\mu, \underline{\theta})\right]=\frac{c}{c+b}(a(\bar{\theta})-a(\underline{\theta})) .
$$

Contracted quantity for the high type (equation (7)) and the low type (equation (8)) are

$$
q(\bar{\theta})=\frac{a(\bar{\theta})}{b+c} \text { and } q(\underline{\theta})=a(\underline{\theta}) \frac{1-(1+\lambda) x}{b+c}
$$

where $\lambda=\frac{\beta}{1-\beta} \frac{c}{c+b}$. To insure the low type is served, we impose

$$
(1+\lambda) x \leq 1
$$

If $\mu>0$, the adjustment market is ex post efficient. Welfare is

$$
W_{\mu>0}^{a}=\frac{(a(\theta)-c \mu)^{2}}{2(b+c)} .
$$


If $\mu=0$, the contracted quantity is efficient for the high type, distorted for the low type. Welfare is

$$
W_{\mu=0}^{a}=\frac{\alpha a^{2}(\bar{\theta})+(1-\alpha)(a(\underline{\theta})(1-(1+\lambda) x))^{2}}{2(b+c)} .
$$

Thus, expected welfare $W^{a}$ is

$$
\begin{aligned}
2(b+c) W^{a}= & \beta\left(\alpha \mathbb{E}\left[(a(\bar{\theta})-c \mu)^{2} / \mu>0\right]+(1-\alpha) \mathbb{E}\left[(a(\underline{\theta})-c \mu)^{2} / \mu>0\right]\right) \\
& +(1-\beta)\left(\alpha a(\bar{\theta})^{2}+(1-\alpha)(a(\underline{\theta})(1-(1+\lambda) x))^{2}\right) \\
= & \mathbb{E}_{\theta} \mathbb{E}_{\mu}\left[(a(\theta)-c \mu)^{2}\right]-(1-\alpha)(1-\beta)\left(a(\underline{\theta})^{2}-(a(\underline{\theta})(1-(1+\lambda) x))^{2}\right) .
\end{aligned}
$$

This expression illustrates the properties of the adjustment market: it is ex post efficient, except for the low type when no production failure occurs (probability $(1-\alpha)(1-\beta))$, where it creates an information distortion $\left(a(\underline{\theta})^{2}-(a(\underline{\theta})(1-(1+\lambda) x))^{2}\right)$.

Welfare variation The previous analysis yields

$$
\begin{aligned}
2(b+c)\left(W^{a}-W^{n a}\right)= & \mathbb{E}_{\theta}\left[\mathbb{E}_{\mu}\left[(a(\theta)-c \mu)^{2}\right]-(a(\theta)-c \mathbb{E}[\mu])^{2}\right] \\
& +(1-\alpha)\left(\begin{array}{c}
\left((a(\underline{\theta})-c \mathbb{E}[\mu])^{2}-(a(\underline{\theta})(1-x)-c \mathbb{E}[\mu])\right. \\
-(1-\beta)\left(a(\underline{\theta})^{2}-(a(\underline{\theta})(1-(1+\lambda) x))^{2}\right)
\end{array}\right) .
\end{aligned}
$$

The first term corresponds to the gain from ex post efficiency. The second term corresponds to the difference in information distortions.

The sign of $\left(W^{a}-W^{n a}\right)$ varies with all the parameters of the problem. To simplify the analysis, we fix $\alpha=\frac{1}{2}$ and $c=b$. Therefore $x=\frac{a(\bar{\theta})-a(\underline{\theta})}{a(\underline{\theta})}$. We also have,

$$
\lambda=\frac{\beta}{2(1-\beta)} \Leftrightarrow 1-\beta=\frac{1}{1+2 \lambda} .
$$

$\lambda($.$) is half the odds ratio. It increases with the probability of failure$ $\beta, \lambda(0)=0$ and $\lim _{\beta \rightarrow 1} \lambda(\beta)=+\infty$. As $\lambda$ decreases, the probability of failure decreases, hence the expected value of the information distortion in $W^{a}$ increases.

Since inverse demand and marginal cost are linear, the gain from ex post 
efficiency takes a very simple form:

$\mathbb{E}_{\theta}\left[\mathbb{E}_{\mu}\left[(a(\theta)-c \mu)^{2}\right]-(a(\theta)-c \mathbb{E}[\mu])^{2}\right]=\mathbb{E}_{\theta}[\operatorname{var}((a(\theta)-c \mu))]=c^{2} \operatorname{var}(\mu)$.

Suppose the shock $\mu$ is distributed according to a Bernoulli distribution: $\mu=0$ with probability $(1-\beta)$, and $\mu=\bar{\mu}$ with probability $\beta$, hence $\mathbb{E}[\mu]=$ $\beta \bar{\mu}$. Then,

$$
\frac{\operatorname{var}(\mu)}{a(\underline{\theta})^{2}}=\frac{\beta(1-\beta) \bar{\mu}^{2}}{a(\underline{\theta})^{2}}=\left(\frac{\mathbb{E}[\mu]}{a(\underline{\theta})}\right)^{2} \frac{1-\beta}{\beta}=\frac{y^{2}}{2 \lambda} .
$$

For a given $\lambda$, the value of ex post efficiency increases with (the square of) expected cost $y$.

With these notation and assumptions, we now examine the sign of $\left(W^{a}-W^{n a}\right)$ as a function of $(c, \lambda, x, y)$. We have

$$
\Delta W \stackrel{\text { def }}{=} \frac{2(b+c)\left(W^{a}-W^{n a}\right)}{a(\underline{\theta})^{2}}=\frac{c^{2} y^{2}}{2 \lambda}-x y+\frac{\lambda x}{2} \frac{(2+\lambda x)}{1+2 \lambda} .
$$

As previously mentioned, its first term corresponds to the welfare gain from ex post efficiency. The net welfare loss due to information distortions is the sum of two terms. The negative first term $(-x y)$ captures the interaction between information and production distortions when no adjustment market is present, that shows that the marginal welfare impact of the information distortion (i.e., $\frac{\partial W^{n a}}{\partial x}$ ) is proportional to the expected cost $y$. The second term $\left(\frac{\lambda x}{2} \frac{(2+\lambda x)}{1+2 \lambda}\right)$ is increasing in $x$ : as the information distortion increases, the value of opening the adjustment market to customers increases.

Opening the adjustment market reduces welfare if and only if $\Delta W<0$. Thus, we examine the sign of $\Delta W$. Consider $\Delta W=0$ as a quadratic equation in $y$. Its discriminant is

$$
D=x\left(x-c^{2} \frac{(2+\lambda x)}{1+2 \lambda}\right)
$$

If $c^{2}$ is high enough, $D<0$, hence $\Delta W(y)>0$ : if the efficiency gain is high enough, opening the adjustment market to load curtailment always improves welfare. 
On the other hand, if $D>0, \Delta W(y)=0$ admits two positive roots

$$
y_{1}=4 \lambda(x-\sqrt{D})<y_{2}=4 \lambda(x+\sqrt{D}) .
$$

Thus, $\Delta W(y)<0$ for $y \in\left(y_{1}, y_{2}\right)$, provided $(1-x-y)>0$. This analysis is formalized below:

Proposition 2 Suppose the consumer has private information on her type. If

$$
c^{2}>\frac{1+2 \lambda}{2+3 \lambda} \equiv \gamma(\lambda)
$$

then opening the adjustment market always improve welfare. If

$$
c^{2}<\frac{1+2 \lambda}{2+9 \lambda} \equiv \delta(\lambda)
$$

there exists $x_{\min }<x_{\max }<\frac{1}{1+\lambda}$ such that opening the adjustment market to energy demanders reduces welfare for all $(x, y) \in \mathcal{H}=\left(x_{\min }, x_{\max }\right) \times$ $\left(y_{1}(x), y_{2}(x)\right)$.

Proof. The details of the proof are presented in Appendix $C$.

If the efficiency gain is lower than the threshold $\delta(\lambda)$, there exists a well-defined set $\mathcal{H}$ for which $\Delta W(x, y)<0$. The function $\delta(\lambda)$ is decreasing in $\lambda$. Suppose $\frac{c^{2}}{\lambda}<\delta(\lambda)$. If $x=0$, there is no private information, hence letting customers enter the adjustment market increases welfare. This is true by continuity around $x=0$. If the information distortion is high enough, specifically if $x \geq x_{\min }$, it outweighs the efficiency gain, hence accepting load shedding in the adjustment market reduces welfare. The constraint that the low type customer be served puts an upper bound on the information distortion, specifically $x<x_{\max }$.

Finally, if $\frac{c^{2}}{\lambda} \in[\delta(\lambda), \gamma(\lambda)]$, there exists a set for which $\Delta W(x, y)<0$, although it is not as clearly defined as when $c^{2}<\delta(\lambda)$, hence we do not describe it.

This simple model has shown that, when suppliers have perfect information on consumers, they can exert first-degree price discrimination, which is good in terms of efficiency and bad in terms of fairness. By contrast, when they only have a statistical knowledge on consumers' types, they can just implement second-best discrimination: they propose a menu made of two 
contracts and each consumer chooses the one he prefers. An efficiency loss (for the "bad type") and more fairness (for the "good type") ensue. Between these bounds, it is a matter of trade-off between the efficiency concern and the fairness concern. In some countries, regulators only worry about competition; others are also in charge of consumers' protection.

\section{$5 \quad$ Regulatory issues}

The need to balance the electric power system in real time to manage the non-storability of electricity is the main explanation for vertical integration in the sector, either structurally or contractually. Non-storability also explains the secondary role traditionally given to demand in the physical balancing of the power system. However, the inclusion of Information and Communication Technologies (ICT) in "smart networks"suggests drastic changes in the near term, providing consumers with a more active role, thus potentially making the whole system more efficient ... and raising new challenges for regulators.

Every consumer can be disconnected at low damage for dates, durations and quantities varying with her equipment and preferences. ${ }^{13}$ The barrier was the cost of implementation. The installation of ICT devices for the remote control of consumption equipment by specialized service providers overcomes this obstacle. It is now technically possible to produce large-scale distributed load-shedding. Additionally, to meet environmental constraints and requirements in energy saving, the active participation of consumers is increasingly seen as politically desirable, especially voluntary demand response.

With the new role given to consumers in electricity markets, we have seen that the demand side must be viewed as behaving more strategically. Market rules and regulations must then be designed to accommodate this evolution. Hereafter, we highlight four problems that must be solved by regulators.

\footnotetext{
${ }^{13}$ For an overview on how to implement demand response, see Borenstein et al. (2002). Torriti et al. (2010) give details about demand response experiences in some European countries.
} 


\subsection{Paying for good resold}

In our analysis, we have assumed that the customers pay for the whole quantity $q$ they contract upon. In the second stage of the game, they decide whether to consume electricity or, when energy shortage occurs, to sell a fraction of the Megawatt-hours they have acquired. Purchasing a good before reselling it is common sense ... for economists, but maybe less for lawyers and politicians. For example, the Federal Energy Regulatory Commission in the United States chose a demand-response mechanism forcing Independent System Operators and Regional Transmission Organizations to pay retail customers the full market price for their reduced consumption, without offsetting the purchase price of this reduced consumption. Many academics have pointed out this ruling is inconsistent with economic analysis. ${ }^{14}$ Chao (2010) shows how voluntary load-shedding helps to correct the inefficiency of time unvarying electricity prices, under the condition that consumers pay the same retail price for the electricity they consume and the electricity they resell. ${ }^{15}$ This is the first necessary layer of regulation for demand response: ex ante, the regulatory authority must impose that consumers are paid the adjustment price for not consuming only if they have purchased the energy they resell. ${ }^{16}$ Otherwise, demand response will be obviously excessive since consumers would be paid for selling something obtained for free.

\footnotetext{
${ }^{14}$ On the FERC case, see USCA 2012a, 2012b and 2014. We thank an anonymous referee of the Journal for the information about the recent overturn of the FERC decision by the US Court of Appeals for the D.C. Circuit Court. The Court first invokes legal reasons: "FERC can regulate practices affecting the wholesale market ..., provided the Commission is not directly regulating a matter subject to state control, such as the retail market." Happily for the economist, it also refers to economic reasons: "Alternatively, even if we assume FERC had statutory authority to execute the Rule in the first place, [its Rule] would still fail because it was arbitrary and capricious". And the Court explains that the FERC decision would overcompensate demand response resources because it requires that demand resources be paid the full marginal price plus be allowed to retain the savings associated with the provider's avoided retail generation cost.

${ }^{15}$ As Ruff (2002) says " Normal markets allow consumers to sell what they do not consume as long as they own it, but no rational market pays consumers for not consuming what they do not own, even if they can prove that they would have bought it but didn't. Paying somebody because they might have bought more but didn't is as illogical, unfair, and inefficient as buying the Brooklyn Bridge from somebody who thought about buying it but decided to sell it instead." See also Crampes and Léautier (2012).

${ }^{16}$ On the PJM demand response payment rules, see Hogan (2009).
} 


\subsection{The market power of aggregators}

Thousands, and progressively millions of consumers will have the possibility to intervene in the adjustment market by reducing their demand below the contracted quantity at a price determined by all the bids of reserve producers and ready-to-renounce customers. However, because of their small scale, most customers will not do it individually. They will rather choose to be connected to a remote operator, able to monitor some domestic appliances and industrial machines for given duration, at some important dates where the electric system needs to be rebalanced. Controlling the possibility to disconnect $1 \mathrm{~kW}$ at one million locations gives the equivalent capacity of a nuclear generation plant, with the additional advantage of time and spatial flexibility. ${ }^{17}$ In this aggregation business, there are strong economies of size (scale, scope, density) as well as indirect network externalities (compatibility, technological complementarity, potential for two-sided payment). This means that the supply of load-shedding will most likely be made by a small number of large firms. In all network activities, take-off is a delicate phase, but when successful, the operator can harvest huge rents thanks to its dominant position. Maintaining a minimal dose of competition without impairing the gains of networking is a difficult task that must be prepared beforehand. ${ }^{18}$

\subsection{Use of collected information}

Energy consumers are very heterogenous: depending of their type (industry, business, household), size, location, technical characteristics of appliances and buildings, they consume large or small, regular or irregular quantities, at peak or off-peak periods. Additionally, depending on the equipment they have installed to produce local energy (photovoltaic panels, wind turbines), they can be net demanders or net suppliers of energy on the grid. The problem is that, electricity being non storable, producing $1 \mathrm{MWh}$ has a much higher cost when it must be supplied in one hour than when the consumer needs $100 \mathrm{~kW}$ per hour for 10 hours. Thus, having a good knowledge of consumption profiles allows to install a cheaper and more reliable production

\footnotetext{
${ }^{17}$ The building of a load-reduction "merit order" based on priority service by aggregators is clearly exposed in Oren (2013).

${ }^{18}$ For a good checklist, see Shapiro (1996).
} 
mix. Unfortunately, traditional meters only give information on aggregated consumptions, sometimes for the last 6 months. Thanks to smart meters, load-shedding protocols and, more generally, demand-response programs, large quantities of precise information will be collected from consumption places and processed to be used by energy suppliers and load-shedding aggegators. This raises two questions. One is that consumers will progressively lose the informational advantage we have presented in the former sections; from our results, it is good news for efficiency, and bad news for equity since suppliers will be able to extract more rents from consumers. Regulators will probably have to intervene to limit the market power of energy suppliers, except if we can enter a world of strong competition in retail. The second question is the trading of data collected on consumers' behavior. Will it be legal to sell data on consumption profiles? Will data on profiles be viewed as an essential facility for new entrants? ${ }^{19}$ Again, it is better to prepare regulations before the problem becomes urgent to solve.

\subsection{Bundling}

In our model, customers who want to exert their rights of reducing consumption have a direct access to the adjustment market. Actually, as suggested above, for most consumers load-shedding will be supplied by specialized operators. Then the question is whether energy suppliers could also be load-shedding aggregators. There are good arguments in favor of this solution. One is that the information necessary for proposing a retail contract are very similar to those necessary to decide on energy savings. Forcing two operators to collect the same data separately would be a costly duplication. A second argument is that the conditions for future demand reduction can be negotiated simultaneously with the retail contract, again saving on transaction costs. Forbidding energy suppliers to propose shedding services would clearly be socially counterproductive.

However, antitrust authorities will have to monitor the terms of dual contracts to be sure that no abuse of dominant position will be exerted. To

\footnotetext{
${ }^{19}$ In September 2014, the French Competition Authority ordered GDF SUEZ, a big energy supplier, to grant its competitors access to parts of its database relating to consumers with regulated gas tariffs. The main argument is that the historical database and the marketing resources inherited from GDF's former monopoly status are necessary tools for the new entrants to develop. See details at www.autoritedelaconcurrence.fr/user/standard.php?id_rub=592\&id_article $=2420$
} 
limit the risks of exclusion of firms proposing one single service, the solution is to accept mixed bundling, that is to oblige firms who can propose the two services to offer them both under a stand-alone format and in a dual contract. Since tying the two services is a source of cost savings, the bundling of energy retail and consumption reduction must be evaluated according to a rule of reason by the competition authorities. ${ }^{20}$

The main difficulty for the authority will be, within a tied offer, to disentangle the price of the two services to assess the value of each. ${ }^{21}$ The task of the energy regulator will rather be to guarantee ex ante that all efficient demand responses are possible, whoever the concerned agents (the producer responsible for energy shortage, the energy retailer and the operator in charge of demand reduction can be three separated entities) and that financial flows are cleared in a fair way. ${ }^{22}$

\section{Conclusion}

This article examines the participation of consumers in adjustment markets for electricity, which enable market participants to respond to random supply shocks occurring after quantities have been contracted. Under perfect competition, opening the adjustment market to consumers always increase ex post efficiency, hence welfare, as expected. However, this result is not robust to strategic behavior by consumers who hold private information on their value for electricity. We prove that under such information asymmetry, allowing consumers to enter the adjustment market may reduce welfare. This arises because suppliers limit the information rents they must abandon by proposing inefficient ex ante retail contracts. If the value of ex post efficiency gains due to consumers' participation is low, whereas the information distortion is high, the overall net effect is a welfare decrease.

Demand response will continue to generate a large academic literature. This work can be expanded in at least two directions. First, it will be important to determine empirically the magnitude of private information on demand for different classes of customers. For example, residential users may have highly predictable demand, hence the extent of information asymmetry

\footnotetext{
${ }^{20}$ See Tirole (2005) for an overview on competition policy in tying cases.

${ }^{21}$ See Hogan (2009) on the PJM rules providing incentives for demand response.

${ }^{22}$ Crampes and Léautier (2012) show the financial accounts concerned when rebalancing an energy shortage with a mix of additional production and demand reduction.
} 
may be limited, and their participation in adjustment markets beneficial. On the other hand, industrial or professional users may have more unpredictable demand, hence information asymmetry may be more of an issue.

Second, if, as seems to be the case, a fraction of customers do have private information on their consumption, research will have to expand the analysis presented in this article to a broader - and more realistic - set of contracts, for example, contracts that allow customers to purchase any quantity up to a limit (controlled by their meter) at a fixed unit price, or contracts that enable customers to buy different blocks of power at different prices.

\section{A No demand participation in adjustment}

Denote $q^{*}(\theta, \mu)$ the ex post efficient production, defined by equality of marginal value and marginal cost

$$
P\left(q^{*}(\theta, \mu), \theta\right)=c\left(q^{*}(\theta, \mu)+\mu\right) .
$$

Full differentiation of the above condition yields

$$
\frac{\partial q^{*}}{\partial \mu}=\frac{c^{\prime}}{P_{q}-c^{\prime}}<0 \Leftrightarrow 1+\frac{\partial q^{*}}{\partial \mu}=\frac{P_{q}}{P_{q}-c^{\prime}}>0
$$

since $P_{q}<0$ and $c^{\prime} \geq 0$. The optimal production decreases with the size of the shock $\mu$. However, $\left(q^{*}(\theta, \mu)+\mu\right)$ increases with the shock $\mu$.

Define $\hat{\mu}$ the production shock such that $c\left(q^{*}(\theta, \hat{\mu})+\hat{\mu}\right)=\mathbb{E}_{\mu}[c(q(\theta)+\mu)]$. For $\mu \leq \hat{\mu}, c\left(q^{*}(\theta, \mu)+\mu\right) \leq c\left(q^{*}(\theta, \hat{\mu})+\hat{\mu}\right)$ since $\left(q^{*}(\theta, \mu)+\mu\right)$ and $c($. are increasing. Thus

$$
\begin{aligned}
& P\left(q^{*}(\theta, \mu), \theta\right)=c\left(q^{*}(\theta, \mu)+\mu\right) \leq c\left(q^{*}(\theta, \hat{\mu})+\hat{\mu}\right)=\mathbb{E}_{\mu}[c(q(\theta)+\mu)]=P(q(\theta), \theta) \\
& \Leftrightarrow \quad q(\theta) \leq q^{*}(\theta, \mu) .
\end{aligned}
$$




\section{B Comparative statics on the adjustment market}

Equilibrium:

$$
\begin{aligned}
p_{a}-S_{q}^{\prime}\left(q-q_{a}^{c}, \theta\right) & =0 \\
p_{a}-C^{\prime}\left(q+q_{a}^{s}\right) & =0 \\
q_{a}^{c}+q_{a}^{s} & =\mu
\end{aligned}
$$

Total differentiation:

$$
\begin{aligned}
d p_{a}-S_{q q}^{\prime \prime} d q+S_{q q}^{\prime \prime} d q_{a}^{c}-S_{q \theta}^{\prime \prime} d \theta & =0 \\
d p_{a}-C^{\prime \prime} d q-C^{\prime \prime} d q_{a}^{s} & =0 \\
d q_{a}^{c}+d q_{a}^{s} & =d \mu
\end{aligned}
$$

or

$$
\left[\begin{array}{ccc}
1 & S_{q q}^{\prime \prime} & 0 \\
1 & 0 & -C^{\prime \prime} \\
0 & 1 & 1
\end{array}\right] \times\left[\begin{array}{c}
d p_{a} \\
d q_{a}^{c} \\
d q_{a}^{s}
\end{array}\right]=\left[\begin{array}{c}
S_{q q}^{\prime \prime} d q+S_{q \theta}^{\prime \prime} d \theta \\
C^{\prime \prime} d q \\
d \mu
\end{array}\right]
$$

Determinant of the full system:

$$
\Delta=C^{\prime \prime}-S_{q q}^{\prime \prime}>0
$$

Effects on $p_{a}$ :

$$
\begin{gathered}
d p_{a}=\frac{\left|\begin{array}{ccc}
S_{q q}^{\prime \prime} d q+S_{q \theta}^{\prime \prime} d \theta & S_{q q}^{\prime \prime} & 0 \\
C^{\prime \prime} d q & 0 & -C^{\prime \prime} \\
d \mu & 1 & 1
\end{array}\right|}{\Delta}=\frac{-S_{q q}^{\prime \prime} C^{\prime \prime} d \mu+C^{\prime \prime}\left(S_{q q}^{\prime \prime} d q+S_{q \theta}^{\prime \prime} d \theta\right)-C^{\prime \prime} S_{q q}^{\prime \prime} d q}{\Delta} \\
\frac{\partial p_{a}}{\partial \mu}=\frac{-S_{q q}^{\prime \prime} C^{\prime \prime}}{\Delta}>0 \\
\frac{\partial p_{a}}{\partial \theta}=\frac{C^{\prime \prime} S_{q \theta}^{\prime \prime}}{\Delta}>0 \\
\frac{\partial p_{a}}{\partial q}=0
\end{gathered}
$$


Effects on $q_{a}^{c}$ :

$$
\begin{gathered}
d q_{a}^{c}=\frac{\left|\begin{array}{ccc}
1 & S_{q q}^{\prime \prime} d q+S_{q \theta}^{\prime \prime} d \theta & 0 \\
1 & C^{\prime \prime} d q & -C^{\prime \prime} \\
0 & d \mu & 1
\end{array}\right|}{\Delta}=\frac{C^{\prime \prime} d q+C^{\prime \prime} d \mu-\left(S_{q q}^{\prime \prime} d q+S_{q \theta}^{\prime \prime} d \theta\right)}{\Delta} \\
\frac{\partial q_{a}^{c}}{\partial \mu}=\frac{C^{\prime \prime}}{\Delta}>0 \\
\frac{\partial q_{a}^{c}}{\partial \theta}=-\frac{S_{q \theta}^{\prime \prime}}{\Delta}<0 \\
\frac{\partial q_{a}^{c}}{\partial q}=1
\end{gathered}
$$

Effects on $q_{a}^{s}$ :

$$
\begin{gathered}
d q_{a}^{s}=\frac{\left|\begin{array}{ccc}
1 & S_{q q}^{\prime \prime} & S_{q q}^{\prime \prime} d q+S_{q \theta}^{\prime \prime} d \theta \\
1 & 0 & C^{\prime \prime} d q \\
0 & 1 & d \mu
\end{array}\right|}{\Delta}=\frac{S_{q q}^{\prime \prime} d q+S_{q \theta}^{\prime \prime} d \theta-C^{\prime \prime} d q-S_{q q}^{\prime \prime} d \mu}{\Delta} \\
\frac{\partial q_{a}^{s}}{\partial \mu}=\frac{-S_{q q}^{\prime \prime}}{\Delta}>0 \\
\frac{\partial q_{a}^{s}}{\partial \theta}=\frac{S_{q \theta}^{\prime \prime}}{\Delta}>0 \\
\frac{\partial q_{a}^{s}}{\partial q}=-1
\end{gathered}
$$

\section{Welfare comparison in the linear case}

Recall that conditions (9) and (10) impose $x<\min \left(\frac{1}{1+\lambda}, 1-y\right)$. We first prove that if $c^{2}>\gamma(\lambda)$, the discriminant $D$ is negative for all $x<\frac{1}{1+\lambda}$. Thus, $\Delta W(x, y)>0$ for all admissible $(x, y)$. If $c^{2}<\gamma(\lambda)$, there exists a unique $x_{\min }<\frac{1}{1+\lambda}$ such that $D>0$ for all $x \in\left(x_{\min }, \frac{1}{1+\lambda}\right)$. We then prove that, if $c^{2}<\delta(\lambda), g\left(x_{\min }\right)=1-x_{\min }-y_{2}\left(x_{\min }\right)>0$. Since $g($.$) is$ decreasing and $g\left(\frac{1}{1+\lambda}\right)>0$, there exists a unique $x_{\max } \in\left(x_{\min }, \frac{1}{1+\lambda}\right)$ such that $g(x) \geq 0$ for all $x \in\left(x_{\min }, x_{\max }\right)$. The results then follows.

Consider the second degree equation with respect to $y$ 


$$
\frac{c^{2} y^{2}}{2 \lambda}-x y+\frac{\lambda x}{2} \frac{(2+\lambda x)}{1+2 \lambda}=0
$$

Its discriminant is

$$
D=x\left(x-c^{2} \frac{(2+\lambda x)}{1+2 \lambda}\right) .
$$

Define $h(x)=x-c^{2} \frac{(2+\lambda x)}{1+2 \lambda}$. Observe that $h(0)=-\frac{2 c^{2}}{1+2 \lambda}<0$. A necessary and sufficient condition for $h(x)>0$ for some value of $x$ lower than $\frac{1}{1+\lambda}$ is

$h\left(\frac{1}{1+\lambda}\right)=\frac{1}{1+\lambda}-c^{2} \frac{\left(2+\frac{\lambda}{1+\lambda}\right)}{1+2 \lambda}>0 \Leftrightarrow \frac{1}{1+\lambda}\left(1-c^{2} \frac{2+3 \lambda}{1+2 \lambda}\right) \Leftrightarrow c^{2}<\frac{1+2 \lambda}{2+3 \lambda}$.

Furthermore,

$$
h^{\prime}(x)=1-\frac{\lambda c^{2}}{1+2 \lambda}>0 \Leftrightarrow c^{2}<\frac{1+2 \lambda}{\lambda},
$$

which holds since $\frac{1}{2+3 \lambda}<\frac{1}{\lambda}$ (this is equivalent to $2+3 \lambda>\lambda \Leftrightarrow 2(1+\lambda)>0$ which is true). Thus, $c^{2}<\frac{1+2 \lambda}{2+3 \lambda}$ is a necessary and sufficient condition for the existence of $x_{\min }<\frac{1}{1+\lambda}$ such that $h(x)>0$ for all $x \in\left(x_{\min }, \frac{1}{1+\lambda}\right)$. Specifically,

$$
h\left(x_{\min }\right)=0 \Leftrightarrow x_{\min }=c^{2} \frac{\left(2+\lambda x_{\min }\right)}{1+2 \lambda}=\frac{\frac{2 c^{2}}{1+2 \lambda}}{1-\frac{\lambda c^{2}}{1+2 \lambda}}=\frac{2 c^{2}}{1+2 \lambda-\lambda c^{2}} .
$$

Therefore, if $c^{2}<\frac{1+2 \lambda}{2+3 \lambda}$ and $x \in\left(x_{\min }, \frac{1}{1+\lambda}\right), \Delta W(y)=0$ admits two positive roots

$$
y_{1}(x)=4 \lambda(x-\sqrt{D})<y_{2}(x)=4 \lambda(x+\sqrt{D}) .
$$

Define $g(x)=1-x-y_{2}(x)$. We are looking for values of $x \in\left(x_{\min }, \frac{1}{1+\lambda}\right)$ such that $g(x)>0$. We first prove that $y_{2}(x)$ is increasing:

$$
y_{2}^{\prime}(x)=4 \lambda\left(1+\frac{1}{2 \sqrt{D}} \frac{d D}{d x}\right)
$$

and

$$
\frac{d D}{d x}=x-c^{2} \frac{(2+\lambda x)}{1+2 \lambda}+x\left(1-\frac{\lambda c^{2}}{1+2 \lambda}\right)=\frac{D}{x}+x\left(1-\frac{\lambda c^{2}}{1+2 \lambda}\right)>0
$$

since $x>x_{\min }$ and $c^{2}<\frac{1+2 \lambda}{2+3 \lambda}<\frac{1+2 \lambda}{\lambda}$. Thus, $y_{2}($.$) is increasing and g($.$) is$ 
decreasing. Observe that

$$
\begin{aligned}
g\left(\frac{1}{1+\lambda}\right) & =1-\frac{1}{1+\lambda}-4 \lambda\left(\frac{1}{1+\lambda}+\sqrt{D\left(\frac{1}{1+\lambda}\right)}\right) \\
& =-\frac{3 \lambda}{1+\lambda}-4 \lambda \sqrt{D\left(\frac{1}{1+\lambda}\right)}<0
\end{aligned}
$$

and

$$
\begin{aligned}
g\left(x_{\min }\right) & =1-x_{\min }-4 \lambda x_{\min }=1-(1+4 \lambda) x_{\min }=\frac{1+2 \lambda-\lambda c^{2}-2 c^{2}(1+4 \lambda)}{1+2 \lambda-\lambda c^{2}} \\
& =\frac{1+2 \lambda-c^{2}(2+9 \lambda)}{1+2 \lambda-\lambda c^{2}} .
\end{aligned}
$$

Thus,

$$
c^{2}<\frac{1+2 \lambda}{2+9 \lambda} \Leftrightarrow g\left(x_{\min }\right)>0,
$$

hence there exists a unique $x_{\max } \in\left(x_{\min }, \frac{1}{1+\lambda}\right)$ such that $g\left(x_{\max }\right)=0$.

This yields the result since $\frac{1}{2+9 \lambda}<\frac{1}{2+3 \lambda}$.

\section{References}

[1] Borenstein S., M. Jaske and A. Rosenfeld (2002) "Dynamic Pricing, Advanced Metering, and Demand Response in Electricity Markets", Center for the Study of Energy Markets, WP 105, University of California, October.

[2] Chao H. P. (2010) "Price-responsive demand management for a smart grid world", The Electricity Journal, vol. 23, Issue 1, pp. 7-20, Jan/Feb.

[3] Crampes C. and T.O. Léautier (2012), "Distributed Load-Shedding in the Balancing of Electricity Markets", EUI RSCAS, WP 2012/40, Loyola de Palacio Programme on Energy Policy

[4] Deller, D., M. Giulietti, J.Y. Jeon, G.Loomes, A. Moniche and C. Waddams (2014), " Measuring consumer inertia in energy", ESRC, Centre for Competition Policy, University of East Anglia, idei.fr/doc/conf/eem/conf2014/Catherine Waddams.pdf 
[5] Hogan W. (2009) "Providing Incentives for Efficient Demand Response", Electric Power Supply Association, Comments on PJM Demand Response Proposals, Federal Energy Regulatory Commission, Docket No. EL09-68-000, Oct.

www.hks.harvard.edu/fs/whogan/Hogan_Demand_Response_102909.pdf

[6] Kamat R. \& S. Oren (2002), "Exotic Options for Interruptible Electricity Supply Contracts," Operations Research, Vol. 50, No. 5; 835-850.

[7] Laffont J.J. \& D. Martimort (2002), "The Theory of Incentives: The Principal-Agent Model", Princeton University Press

[8] Oren S.(2013), "A Historical Perspective and Business Model for Load Response Aggregation Based on Priority Service", 46th Hawaii International Conference on System Sciences, http://www.ieor.berkeley.edu/ oren/pubs/I.B2.189.pdf.

[9] Ruff L. (2002) "Economic principles of demand response in electricity", Edison Electric Institute, Washington DC, Oct.

[10] Shapiro C. (1996), "Antitrust in network industries", Address before the American Law Institute and American Bar Association, www.justice.gov/atr/public/speeches/0593.pdf

[11] Stole L. A. (2007) "Price Discrimination in Competitive Environments", Handbook of Industrial Organization, Vol. 3.

[12] Tirole J. (2005). "The Analysis of Tying Cases : A Primer", Competition Policy International, vol. 1, n 1, Spring.

[13] Torriti J., M. G. Hassan and M. Leach (2010), "Demand response experience in Europe: Policies, programmes and implementation", Energy, Volume 35, Issue 4, pp. 1575-1583.

[14] USCA (2012a), "Economists' Brief on FERC Order 745 regarding demand response compensation", Amici Curiae Brief, District Court of Columia Circuit, Electric Power Supply Association, June, Case \#111486, Document \#1378605

www.hks.harvard.edu/hepg/Papers/2012/Economists amicus brief_061312.pdf 
[15] USCA (2012b), "Reply brief of petitioneers", October, Case \#11-1486, Document \#1399196

www.publicpower.org/files/PDFs/ReplyBriefEPSAvFERC10112012.pdf

[16] USCA (2014), "Electric Power Supply Association v. FERC", May, Case \#11-1486, https://energylawprof.files.wordpress.com/2014/06/epsa-v-ferc.pdf

[17] Wilson, C.M. and C. Waddams Price (2010), "Do consumers switch to the best supplier?", Oxford Economic Papers 62, pp. 647-668 